JPHSR 2011, 2: 175-184

(C) 2011 The Authors

JPHSR @ 2011 Royal

Pharmaceutical Society

Received August 17, 2010

Accepted June 8, 2011

DOI

10.1111/j.1759-8893.2011.00057.x ISSN 1759-8885
Correspondence: Morro M.L. Touray, Health Economics and Policy Research Unit, Faculty of Health, Sport and Science, University of Glamorgan, Pontypridd CF37 1 DL, UK. E-mail:

mmltouray@alumni.Ise.ac.uk; mtouray@glam.ac.uk

\section{The cost effectiveness of pneumococcal conjugate vaccine in the routine infant immunisation programme of The Gambia}

\author{
Morro M.L. Touray ${ }^{a}$, Raymond Hutubessyc and Arnab Acharya ${ }^{b}$ \\ aUniversity of Glamorgan, Pontypridd, UK, 'bondon School of Hygiene and Tropical Medicine, London, \\ UK and Immunization, Vaccines and Biologicals (IVB), WHO, Geneva, Switzerland
}

\section{Abstract}

Objectives To evaluate the cost effectiveness of the use of nine-valent pneumococcal polysaccharide conjugate vaccine in a routine infant immunisation programme based on the Pneumococcal Vaccine Trial (PVT) study in The Gambia.

Methods This was a clinical trial-based cost-effectiveness study conducted as part of the PVT study. The PVT was an intention-to-treat double-blind placebo-controlled trial of a nine-valent pneumococcal polysaccharide conjugate vaccine. The trial was conducted in the eastern parts of The Gambia, West Africa and recruited 17437 children aged 40-364 days. A deterministic static cohort model was developed to evaluate direct benefits and costs of pneumococcal conjugate vaccine in The Gambia's routine immunisation programme. The incremental cost-effectiveness ratio (iCER) is defined as vaccinating infants against pneumococcal disease compared with no vaccination from a public provider's perspective using The Gambia's 2005 projected under-one-year population.

Key findings The results show the use of the vaccine in The Gambia's routine infant vaccination programme to be cost effective using an assumed price of US\$5.00 per vial in single-dose vials. Compared with offering no vaccination, the incremental cost per DALYs averted would be 30 DALYs from the public provider perspective. At least 1569 and 340 invasive childhood pneumococcal illnesses and deaths respectively among the cohort would be prevented. In the absence of the vaccine 16871 DALYs would be lost while with the use of the vaccine 7804 DALYs would be lost. Given the average treatment cost of pneumococcal illnesses to be US\$191 (95\% confidence interval 180 to 203) the introduction of the vaccine programme would lead to an additional cost of US\$274 279 (about US\$8.43/child). Conclusions The availability of a cost-effective vaccine that can prevent thousands of pneumococcal illnesses and related deaths is a major development towards improving the disease burden in sub-Saharan African countries. This study supports the introduction of nine-valent pneumococcal vaccine into the infant immunisation programme of The Gambia as it is cost effective and will avert many preventable deaths and illnesses. Despite differences in distribution of serotypes between countries, the static model used in the analysis makes the results applicable to other developing countries, particularly those in sub-Saharan Africa.

Keywords cost effectiveness; developing countries; economic evaluations; low- and middle-income countries; pneumococcal conjugate vaccine; The Gambia

\section{Introduction}

Diseases caused by Streptococcus pneumoniae are a major public health problem worldwide. Illnesses that are often caused by pneumococci include pneumonia, meningitis and febrile bacteraemia; otitis media, sinusitis and bronchitis are a more common but less serious manifestation of infection. Pneumonia is estimated to be responsible for about 1.6 million deaths around the globe every year and most of these are caused by pneumococcus bacteria. $^{[1,2]}$ It is documented that it is the highest cause of mortality and morbidity in children around the world, more than malaria, tuberculosis and HIV/AIDS combined. ${ }^{[3]}$ In subSaharan Africa the incidence of pneumococcal disease is almost 10 times higher than in developed countries. ${ }^{[2]}$

Evidence from The Gambia suggests causes of severe pneumonia-related illnesses among children admitted to Gambian hospitals, and related deaths due to acute lower respiratory infections, can be attributed to pneumonia. ${ }^{[4,5]}$ Against this background, coupled with 


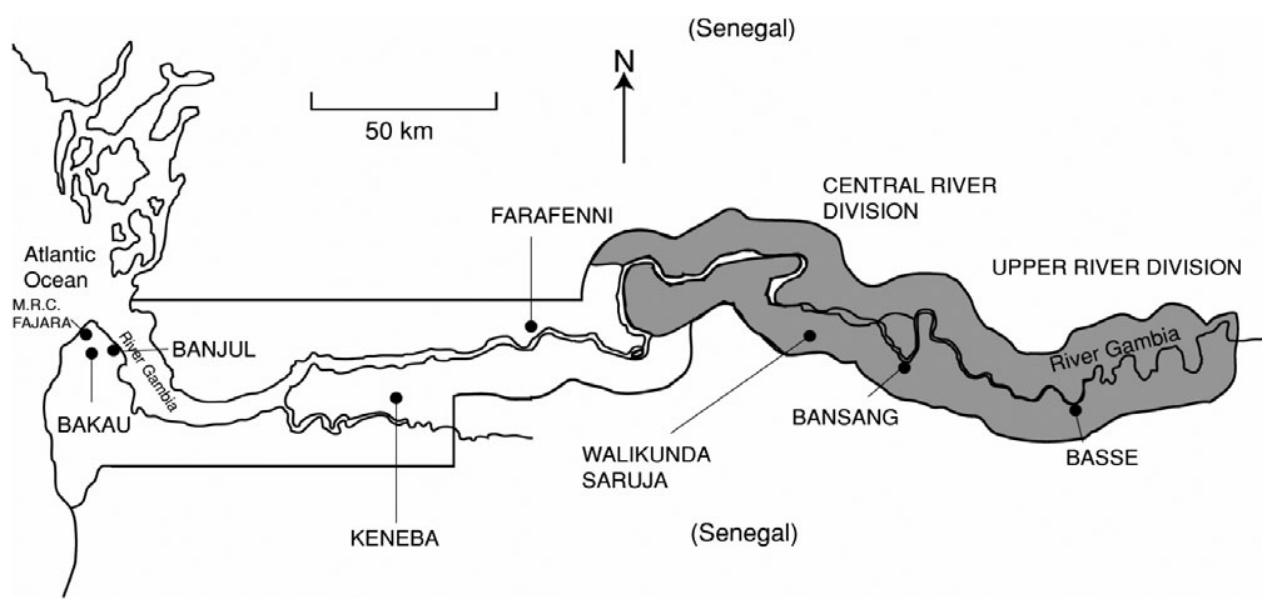

Figure 1 Map of The Gambia showing the Pneumococcal Vaccine Trial study area (shaded).

findings that have shown conjugate vaccines to be safe, immunogenic and to induce immunological memory in Gambian infants, a 5-year study to assess the efficacy of nine-valent pneumococcal polysaccharide/protein conjugate vaccine against radiological pneumonia was initiated as hosted by the UK Medical Research Council in collaboration with the Government of The Gambia. The Pneumococcal Vaccine Trial (PVT) study was a double-blind (intention-to-treat) randomised placebo-controlled trial with the primary objective to determine the efficacy of nine-valent pneumococcal polysaccharide/protein conjugate vaccine in reducing incidence of X-ray diagnosed radiological pneumonia. The study was conducted in the eastern parts of The Gambia covering two administrative areas of Central and Upper River Divisions (Figure 1). The trial recruited 17437 children, aged 40 to 364 days, in the two administrative divisions.

The study showed the vaccine to be $37 \%$ (95\% confidence interval (CI) 27 to 45) efficacious against pneumococcal disease (all causes) and $77 \%$ efficacious against illnesses attributable to serotypes present in the vaccine with a 95\% CI of 51 to 90 in per protocol analysis. ${ }^{[6]}$ The vaccine covers about $75 \%$ of serotypes that cause infections in Gambian children.

According to a recent health policy paper by the Gambian Ministry of Health (MOH) ${ }^{[7]}$ Gambia plans to expand its vaccination programme both in terms of coverage and introduction of new essential vaccines such as the pneumococcal conjugate vaccine. Although a seven-valent pneumococcal vaccine (Prevenar), which covers fewer serotypes, has been introduced into the routine infant vaccination programme with the assistance of the Global Alliance for Vaccines and Immunisation (GAVI) (a global health partnership between private and public sectors for the protection of people's health by increasing access to immunisation in poor countries and other international donors), questions on the economic feasibility of introducing the vaccine vis-à-vis the continuation of provision of treatment of pneumococcal diseases have not been answered. This paper reports on the projected effects, costs and cost effectiveness of nine-valent conjugate pneumococcal vaccination using specific PVT clinical study data ${ }^{[6]}$ in order to help the decision making process regarding the adop- tion and scaling up of pneumococcal vaccination in The Gambia. The GAVI is also considering supporting other subSaharan countries to introduce the vaccine and the paper will certainly be helpful in that consideration process.

\section{Methods}

\section{Analytic overview}

A computer based decision analysis model using Microsoft Excel and Tree AgePro 2008 (Figure 2) was developed to follow a birth cohort of 32544 Gambian infants born in 2005 following standard methods for economic evaluations of immunisation programmes ${ }^{[8-12]}$ to assess lives saved, disability adjusted life years (DALYs) averted, costs and cost effectiveness of nine-valent pneumococcal conjugate vaccination of infants. The two strategies examined in the cost effectiveness analysis are 'vaccinations' i.e. the purchase and provision of the pneumococcal vaccine and treatment against "no vaccinations' i.e. treatments only which reflects the status quo before the introduction of Prevenar vaccine. Vaccine costs and the Expanded Programme on Immunisation (EPI) and treatment costs for long-term sequelae were included in the costs analysis while indirect costs incurred by households were not taken into consideration. The efficacy is based on the clinical trial estimates as reported by Cutts et al. ${ }^{[6]}$

The analysis was carried out from a public health provider perspective. The PVT's budgets were provided and allocated in US dollars and costs used in all the calculations in this analysis are reported in the same currency. Future costs and outcomes were discounted at $3 \%$ per year while we adjusted for inflation using the Gambian gross domestic product (GDP) deflators ${ }^{[13]}$ and expressed costs in 2005 US\$.

The cost effectiveness of a programme was benchmarked in reference to the World Health Organization (WHO)'s Commission on Macroeconomics and Health ${ }^{[14]}$ which classifies interventions as 'highly cost effective' for a given country if results show that they avert a DALY for less than the per capita national GDP, 'cost effective' if one-to-three times GDP per capita and 'not cost effective' if it is greater than three times GDP per capita. The affordability at country level 


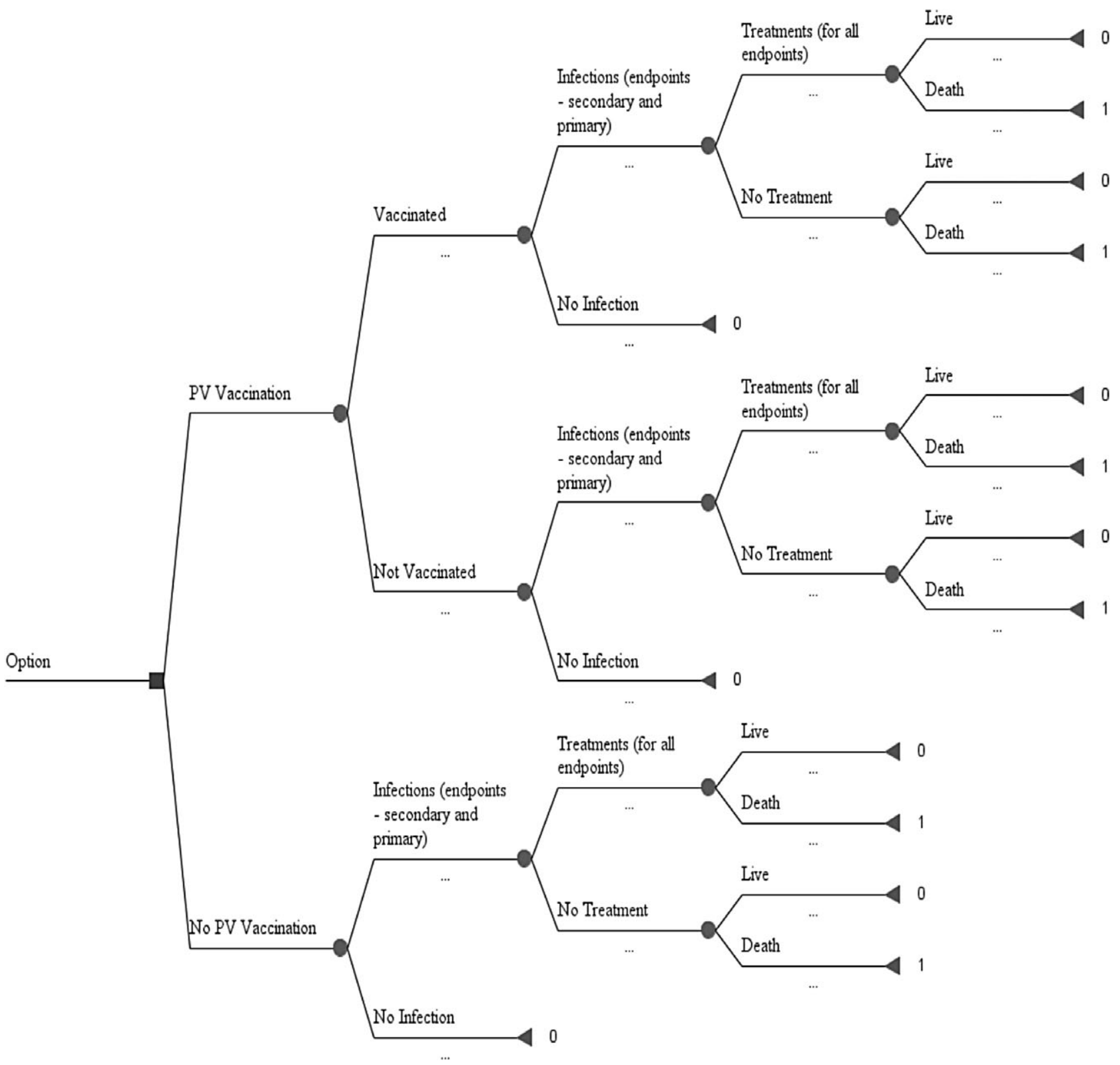

Figure 2 The decision tree: a computer based decision analysis model using Microsoft Excel and Tree AgePro 2008.

was assessed in terms of the annual expected cost for the vaccination programme compared with the national health expenditure for 2005 .

To explore parameter uncertainty we conducted one-way and multivariate sensitivity analyses. Analyses were done in Microsoft Office Excel 2003 and StataSE release 9 while TreeAge Pro 2008 was used for the decision tree and to structure the analytical model (Figure 2).

\section{Costs}

We used a conservative price of US\$5 per single dose vial based on the 2007 assumptions of the Advanced Market Commitment (AMC) - a funding mechanism to stimulate the development and manufacture of new vaccines for developing countries. ${ }^{[15]}$ Three doses are required for a complete course at intervals of at least 25 days between doses. Vaccine programme costs were estimated under the assumption that pneumococcal vaccination would be incorporated into routine vaccine administration during infancy. Estimation of the country's infant vaccination programme under the EPI of The Gambia's Ministry of Health and vaccination costs were based on the financial sustainability plan (FSP) for The Gambia provided to the GAVI in $2003^{[16]}$ which includes freight; storage and distribution costs; additional cold chain requirements for storage and transportation; injection materials; time spent on patient education; publicity to introduce the vaccine; health workers' training; and dealing with adverse effects of the vaccine. Surveillance costs have also been incorporated based on FSP and EPI projections for the vaccine's introduction. The FSP is a document that assesses financing 
Table 1 Model variables, assumptions and data sources

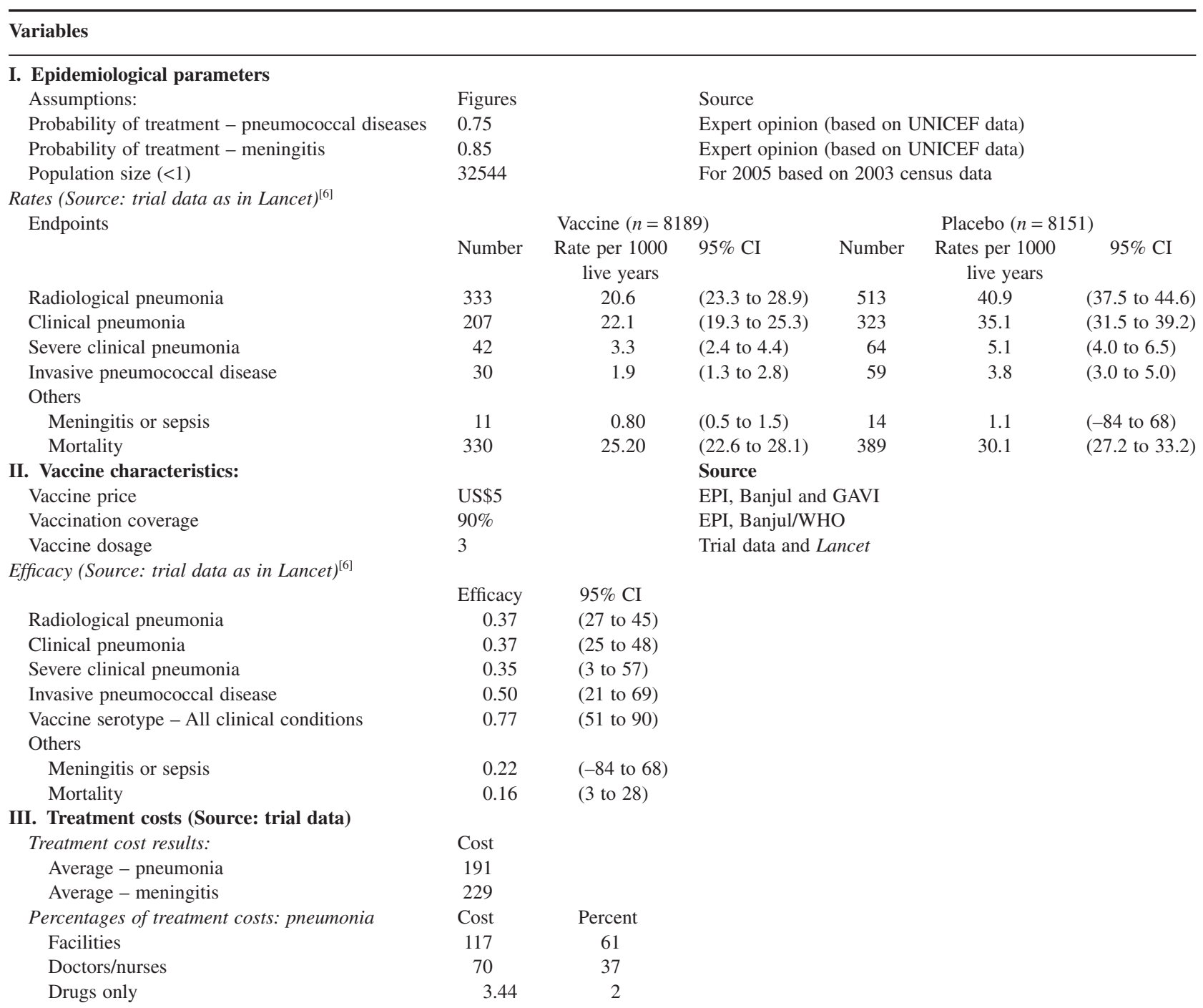

CI, confidence interval; EPI, Expanded Programme on Immunization; GAVI, Global Alliance for Vaccines and Immunisation; $n=$ number of cases in the trial. All costs/prices are expressed in 2005 US\$.

challenges facing a national immunisation programme. ${ }^{[17]}$ It provides projections of resources requirements for the national immunisation programme hence describing the government's approach to mobilising and effectively using financial resources to support medium and long-term programme objectives. ${ }^{[17]}$

Treatment costs include physicians/nurses fees, drugs, diagnosis and testing costs, facilities and related costs (including inpatients' and carers' feeding). Prospective data collections for treatment cost analysis started in early 2003 and continued until the PVT study data was locked in March 2004. Hence the cost-effectiveness analysis includes cost of treating all study subjects (both in- and outpatients) that were diagnosed with the trial endpoints namely radiological pneumonia, clinical pneumonia, severe clinical pneumonia, invasive pneumococcal disease and meningitis/sepsis during this period. Drugs and associated medical items used for treatment were obtained from the trial's patient-level database $\mathrm{e}^{[6]}$ complemented with prices from the International Drug Price Indicator Guide ${ }^{[18]}$ in cases where prices could not be traced from trial records. The costs for hospital days and staff time including physician and nurse times were derived from the WHO-CHOICE data for Gambia. ${ }^{[16]}$ Diagnosis (i.e. chest $\mathrm{X}$-ray) costs were based on Drug Revolving Fund charges available in Gambian hospitals (obtained through MRC's Radiology Unit).

\section{Probabilities on healthcare access and vaccine coverage}

Table 1 provides information on relevant epidemiological data and assumptions. Where children have access to health facilities, availability of appropriate treatment at the facility cannot be guaranteed. Since information is not available on this and some other areas, a number of assumptions were 
made. UNICEF's specific country data on The Gambia indicate that $85 \%$ of children are taken to health facilities when they have acute lower respiratory infections. Data were obtained from UNICEF's websites: childinfo.org, Immunization Coverage Table 2004 (accessed 12 August 2005) and http://www.unicef.org, Health Statistics 2005 (accessed 12 August 2005). We therefore used these figures to represent the probability of treatment for meningitis cases and slightly lower figures of $75 \%$ for the primary and secondary endpoints. With these assumptions, from a cohort of about 32544 under one year of age population a total of 867 (including one possible meningitis case) are expected to be treated if the vaccine is introduced compared to 2075 (including three possible meningitis cases) in the absence of the vaccine.

A number of publications ${ }^{[19,20]}$ along with The Gambia's EPI office, put the yearly complete vaccination coverage at $90 \%$. We therefore used $90 \%$ as the probability of vaccination for the static model used. Morton ${ }^{[21,22]}$ indicated that at least $75-85 \%$ of patients with meningitis in the USA before the antibiotics era would not have survived. Based on expert opinion (from PneumoAdip, through personal communications with Dr O. Levin) for the model used here, case fatalities for various illnesses when untreated are the following: $90 \%$ for meningitis, with lower figures of $75 \%$ for the primary and secondary endpoints. We are aware that there is about $8 \%$ probability of resistance to antibiotics based on studies and research conducted in The Gambia and other countries. ${ }^{[23-27]}$ Where treatment is provided we used all-cause mortality rate for the vaccine as in Table 1. Regarding probabilities of permanent disabilities of deafness, learning difficulties, motor deficit and seizure disorders, the proportions of permanent disabilities following meningitis treatment in a study by Tessa et al. ${ }^{[28]}$ were used. The results of this same study were used in our estimation of probability of recovery from meningitis following treatment.

\section{Efficacy}

The trial's efficacy results as published in Cutts et al. ${ }^{[6]}$ were used in the estimation of effects for all conditions. We report vaccine efficacy (VE) as a summary of the trial in Table 1. However, some incident and mortality rates used in calculating certain probabilities were derived from other studies as explained above.

\section{Outcome measures}

We compared the options of vaccinating the cohort of children aged 40-364 with that of no vaccination where all cases of illnesses are assumed to occur between the ages of 1-4 years. We measured incremental cost as:

\section{Incremental cost $=($ cost of vaccination + cost of treating illnesses among the vaccination cohort) - (cost of treatment among the no vaccination cohort)}

The benefits are expressed in terms of prevented cases, mortality and DALYs related to pneumococcal-related illnesses. DALYs are the sum of years of present value of future life lost due to premature mortality and the years of future productive life lost due to disability as a result of an illness. Our calculation of DALYs were based on the global burden of disease (GBD) formula (Murray et al. $)^{[29]}$ and the disability weights as well as age weighting were also from the GBD formula. The incremental benefits are defined as:

$$
\begin{aligned}
\text { Incremental benefit }= & (\text { DALYs accruing to the cohort } \\
& \text { without the vaccinations })-(\text { DALYs } \\
& \text { accruing to the cohort with } \\
& \text { vaccinations }) .
\end{aligned}
$$

The incremental cost-effectiveness ratio (iCER) is the ratio of incremental cost and incremental benefit.

\section{Uncertainty and multivariate sensitivity analysis}

In the base case analysis a vaccine price of US\$5 was used $^{[15]}$ with a vaccine efficacy of $37 \%$ as in Table $1^{[6]}$ and a vaccination coverage of $90 \% .^{[20]}$ The overall vaccine efficacy, vaccination coverage and the price of the vaccine were subjected to one-way and multivariate probabilistic sensitivity analyses to assess their impacts on the model and various outcomes.

A per-dose cost of US\$65 was used as an upper bound estimate of the vaccine price (in the one-way sensitivity analysis) as during the trial seven-valent Prevnar was supplied at this price for vaccination of trial controls. While anticipating a cap of US\$3.5 per dose during the tail period of the AMC (Reference: http://www.vaccineamc.org) ${ }^{[30]}$ we, however, used US\$1 as the lower bound estimate in the sensitivity analysis and US\$35 as the worst-case price in the multivariate sensitivity analysis. The lower and upper efficacy estimates were $40 \%$ less and $100 \%$ more respectively to bring them near the lower limit $\mathrm{CI}$ for $\mathrm{VE}$ and the VE figure for vaccine serotypes only as in Table 1.

The Gambia's EPI registered different coverage rates for various antigens used in the programme from 2000 to 2005. These range from $75 \%$ (lowest) for Hib and DTP3 to 100\% (the highest) for BCG. For the multivariate sensitivity analysis purposes, we used the lowest and highest coverage figures for the period as our lower and upper bound estimates respectively.

\section{Results}

\section{Illnesses, deaths and disabilities averted}

From the cohort of 32544 , without the vaccine our model anticipates 2763 pneumococcal-related illnesses whilst with the use of the vaccine there would be 1194 possible invasive pneumococcal infections. Thus the new programme would result in the reduction of 1569 infections. The illnesses averted include radiological pneumonia, clinical pneumonia, severe clinical pneumonia, invasive pneumococcal disease and meningitis/sepsis.

The reduction in incidence of illnesses results in averting a total of 340 deaths. Some of those surviving the illnesses would have long-term disabilities particularly in meningitis cases. Assuming the disabilities to be from only meningitis cases, the model anticipates insignificant cases of permanent 
disabilities. This is due to the insignificant meningitis cases realised during the clinical trial. The disabilities taken into account were deafness, severe autism, motor-deficit and seizure disorders.

\section{Disability adjusted life years averted}

The loss of DALYs with and without the use of the vaccine in the national infant vaccination programme stand at 7804 and 16871 respectively implying 9067 DALYs averted with the use of the vaccine.

\section{Costs}

The overall average cost of treatment was calculated to be US\$191 (95\% CI 180 to 203). The lowest treatment cost was US\$47 and the highest was US\$1815. The average medical treatment cost of drugs only was US\$3.44 (95\% CI 3 to 4) with US\$0.05 as lowest and the highest US\$60 (Table 2). The main drivers of treatment costs were facilities and medicalstaff associated costs, accounting for $61 \%$ and $37 \%$ respectively of overall average treatment cost.

The cost analysis component of the trial involved 677 study participants. All study participants admitted for treatment or attended to as outpatients had co-morbidities including our diagnosis of interest. For such co-morbidity cases, resources used in all the respective treatments have been accounted for in the estimation of treatment costs. Although a large number of those with co-morbidities were given nutritional supplements for malnutrition, nutritional supplements were however not included in our treatment cost estimations.

The cost of nine-valent vaccine alone with $15 \%$ wastage rate based on the 2005 projected under one-year population would be US\$561 384 (using three doses per child). When all other costs are taken into account, introduction of the vaccine would induce a cost of US\$1 976537 . The cost would be incurred by the EPI when delivered through the routine programme and by the rest of the health system due to the necessity of treating any cases of illnesses arising even with the vaccine. With the status quo of only the provision of treatment for childhood pneumonia and related cases, the cost is estimated to be US\$1 702258. The two estimates yield an incremental cost of US\$274 279. Using the 2005 population projections there would be an additional cost of US\$8.43 for each child aged under one year.

As there were very few meningitis cases in our study, we adjusted the average treatment cost by $20 \%$ (following consultation with the Trial Paediatricians) to represent average meningitis treatment cost. Meningitis patients would likely spend more time in hospital than pneumococcal pneumonia patients. Table 3 illustrates various cost differences between the two options. Due to the insignificant meningitis cases, costs associated with meningitis treatment have no influence on the model.

\section{Incremental cost-effectiveness ratios}

As for the case of programme and vaccine-related costs presented above, the incremental cost-effectiveness ratios (iCERs) also varied significantly depending on vaccine price. Using the presumed price of US\$5.00 per vial in single-dose vials the iCERs were 175,807 and 30 for pneumococcal illnesses, deaths and DALYs averted respectively (Table 3).

Table 2 Average cost of treatment

\begin{tabular}{|c|c|c|c|c|c|c|}
\hline & \multicolumn{3}{|c|}{ Average } & \multicolumn{3}{|c|}{ 95\% Confidence interval } \\
\hline & Average & Low & High & Low limit & Upper limit & Standard deviation \\
\hline Bed days (length of stay) & 3.45 & 1 & 33 & 3 & 4 & 3 \\
\hline Medical treatment costs (drugs only) & US $\$ 3.44$ & US\$0.05 & US\$59.82 & 3 & 4 & 5 \\
\hline Facilities costs only & US\$116.53 & US\$25.10 & US\$1131.59 & 109 & 124 & 97 \\
\hline Doctors/nurses (staff) Costs only & US $\$ 69.88$ & US\$20.27 & US\$668.88 & 66 & 74 & 57 \\
\hline Medical treatment costs (Drugs and staff) & US\$74.75 & US $\$ 21.75$ & US\$683.64 & 70 & 79 & 59 \\
\hline Overall treatment costs & US\$191.28 & US\$47.29 & US\$1815.23 & 180 & 203 & 156 \\
\hline
\end{tabular}

All costs/prices are expressed in 2005 US\$.

Table 3 Cost effectiveness results and cost differences

\begin{tabular}{|c|c|c|c|c|}
\hline & No $P V$ vaccination option & PV vaccination option & Incremental cost or effect & iCERs \\
\hline Overall costs & US\$1 702258.00 & US\$1 976536.65 & US\$274 278.65 & \\
\hline Illnesses & 2763 & 1194 & 1569 & 175 \\
\hline Deaths & 582 & 242 & 340 & 807 \\
\hline DALYs lost & 16871 & 7804 & 9067 & 30 \\
\hline Treatment costs & US\$396968.93 & US\$166002.43 & -US\$230966.50 & \\
\hline Illnesses & 2763 & 1194 & 1569 & 147 \\
\hline Deaths & 582 & 242 & 340 & 679 \\
\hline DALYs & 16871 & 7804 & 9067 & 26 \\
\hline
\end{tabular}

iCER, incremental cost-effectiveness ratios; PV, pneumococcal vaccine; negative value = cost-savings. All costs/prices are expressed in 2005 US\$. 


\section{Uncertainty and sensitivity analysis}

The model shows that VE is proportional to both incremental costs and incremental effects (of illnesses, deaths and DALYs averted). When VE is $74 \%$ there would be an incremental cost of US\$242 169. At VE of $90 \%$ and $100 \%$ there would be incremental costs of US\$228 041 and US\$219 693 respectively. With regards to the incremental effects, VE is directly proportional to DALYs saved, illnesses and deaths averted. As vaccination coverage increases, it influences both health outcomes and costs. At $95 \%$ and $100 \%$ coverage, there would be incremental costs of US\$289 549 and US\$304 820 respectively.

Subjecting price to sensitivity analysis and using vaccine prices of US\$1.00 and US\$2.00 (all others remaining

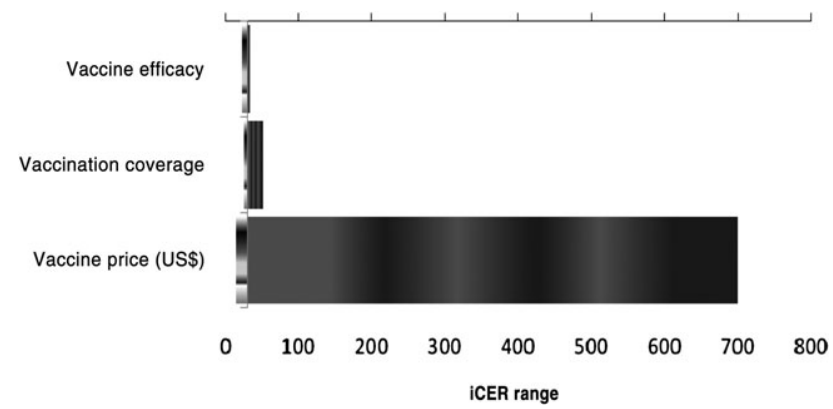

Figure 3 Tornado diagram showing the influence of price, vaccine efficacy and coverage on incremental cost-effectiveness ratio and disability-adjusted life years. $(\equiv$ Best case; $(\square)$ Worst case. unchanged) there would be cost-savings of US\$129917 and US\$28 868 respectively. Vaccine price is inversely proportional to cost-savings and using a straight-line equation, while other variables remain unchanged, incremental cost is zero at vaccine price of about US\$2.28.

With VE and vaccination coverage being proportional to both health outcomes and costs, lowering the coverage would certainly have negative impacts. At the price of US\$65.00, the use of the vaccine would increase the cost of a vaccination option to US\$8 039479 (other factors remaining unchanged) representing an additional cost of US\$6 337221 to the EPI's budget for introducing the vaccine. The tornado diagram in Figure 3 shows the influence of price, vaccine efficacy and coverage on iCERs (DALYs).

A number of variables were subjected to multivariate uncertainty analysis. We adopted base, worst and best case scenarios for the multivariate uncertainty analysis along vaccine price. The baseline scenario is based on trial results and the average national vaccination coverage. The worst and best case scenarios mimic the lower limit confidence intervals for VE (radiological pneumonia) and the VE for vaccine serotypes only. In the case of vaccination coverage the lowest and highest coverage registered for various antigens by the EPI for the period 2000-2005 were used. Using VE, vaccination coverage and vaccine price of $22 \%$, $75 \%$ and US\$35 respectively, the impact on the EPI budget would be an additional sum of US\$3 318 593. Multivariate sensitivity analysis (Table 4) also indicates that all the outcomes but iCER - DALYs are directly proportional to both vaccination coverage and VE. At vaccine price of US\$1, there would be cost-savings of US\$129917 (US\$117074

Table 4 Multivariate sensitivity analysis

\begin{tabular}{|c|c|c|c|}
\hline & Worst case & Baseline & Best case \\
\hline \multicolumn{4}{|l|}{ A. Baseline price of US $\$ 5$} \\
\hline Coverage & $75 \%$ & $90 \%$ & $100 \%$ \\
\hline Efficacy & $22 \%$ & $37 \%$ & $74 \%$ \\
\hline iCER - DALYs & 34 & 30 & 23 \\
\hline iCER - Deaths averted & 894 & 807 & 626 \\
\hline iCER - Illnesses averted & 194 & 175 & 135 \\
\hline Incremental cost $(-\mathrm{Ve}=$ cost-savings $)$ US $\$$ & US\$287 122.00 & US\$274 278.65 & US\$242 169.00 \\
\hline \multicolumn{4}{|l|}{ B. Worst case price US $\$ 35$} \\
\hline Coverage & $75 \%$ & $90 \%$ & $100 \%$ \\
\hline Efficacy & $22 \%$ & $37 \%$ & $74 \%$ \\
\hline iCER - DALYs & 392 & 365 & 310 \\
\hline iCER - Deaths averted & 10336 & 9728 & 8467 \\
\hline iCER - Illnesses averted & 2243 & 2107 & 1827 \\
\hline Incremental cost $(-\mathrm{Ve}=$ cost-savings $)$ US $\$$ & US\$3 318593.00 & US\$3 305750.00 & US\$3 273640.00 \\
\hline \multicolumn{4}{|l|}{ C. Best case price of US\$1 } \\
\hline Coverage & $75 \%$ & $90 \%$ & $100 \%$ \\
\hline Efficacy & $22 \%$ & $37 \%$ & $74 \%$ \\
\hline iCER - DALYs & 14 & 14 & 15 \\
\hline iCER - Deaths averted & 365 & 382 & 419 \\
\hline iCER - Illnesses averted & 79 & 83 & 90 \\
\hline Incremental cost $(-\mathrm{Ve}=$ cost-savings $)$ US $\$$ & $\begin{array}{l}\text {-US\$117 } 074.00 \\
\text { Efficacy reduced by } 40 \% \\
\text { Coverage reduced to } 75 \%\end{array}$ & -US\$129917.00 & $\begin{array}{l}\qquad-\mathrm{US} \$ 162027.00 \\
\text { Efficacy increased by } 100 \% \text { to near } 77 \% \\
\text { for efficacy against vaccine serotypes } \\
\text { Coverage increased to } 100 \%\end{array}$ \\
\hline
\end{tabular}

DALY, disability-adjusted life years; iCER, incremental cost-effectiveness ratios; -Ve, negative value. All costs/prices are expressed in 2005 US\$. 
and US\$162027 in the worst and best case scenarios respectively).

\section{Discussions}

The Gambia does not have local cost-effectiveness guidelines or a cost-effectiveness league table to use as a yardstick for this analysis. However, using WHO's Commission on Macroeconomics and Health classification of cost-effectiveness interventions and the Guide on Standardisation of Economic Evaluations of Immunisation Programmes ${ }^{[1,31]}$ the use of the vaccine is no doubt highly cost effective. Moreover, for 2227 illnesses, a possible 340 deaths and 9067 DALYs would be averted with the use of the vaccine, all at additional cost of US $\$ 274279$; the evidence that the outcomes of using the vaccine far outweigh the provision of treatment alone cannot be overstated.

However effective and efficient a preventive programme might be, affordability of the vaccine is an essential issue particularly for developing countries. In that light vaccine costs and prices continue to be essential budget items in vaccination programmes. At certain prices, the vaccine would not only prove to be expensive for low and middle-income countries like The Gambia but the cost effectiveness (in the context of prevention versus treatment) would be discredited. For example when the price of the vaccine is US\$65.00 (as was supplied during the vaccination of trial controls), it would be economically logical, at least in the context of the burden on the EPI's budget, to go for the treatment option since the additional cost of the vaccine alone would be more than the whole EPI programme (including costs of vaccines in use before Prevenar's introduction). Here, there would be an additional cost of over US\$6.3 million which in combination with the public health care financing structure in The Gambia (which is over $80 \%$ donor driven) poses challenges regarding sustainable and affordable implementation of the vaccine into the routine immunisation schedule.

The base-case analysis of our study has the following limitations. Firstly our study did not credit for herd immunity protection. Several studies in high-income countries have shown indirect effect of routine pneumococcal vaccination, i.e. large decreases in invasive pneumococcal disease in unvaccinated children. ${ }^{[32,33]}$ Secondly, possible serotype replacement has not been factored. Serotype replacement, a situation in which declines in diseases due to vaccine-type pneumonia are counterbalanced by increases in diseases caused by serotypes not covered by the vaccine, has the potential to limit (or relatively reduce) the impact of the vaccine. ${ }^{[34]}$ Many studies reported evidences of serotype replacements ${ }^{[32,34-37]}$ and this can be a concern in that its resultant limitations on the impact of the vaccine would not be mitigated by revaccination. Serotype replacements therefore indicate a potential need for the vaccine's reformulations and new economic evaluations to account for serotype replacements as well as other variables such as time (period after vaccination). Thirdly, the study ignored indirect costs related with PCV vaccination such as time and productivity losses of parents and guardians due to pneumococcal disease. Inclusion of these costs would have made the programme more attractive from a cost-saving perspective. Finally since the risk of adverse effects of the vaccine are very low $^{[6]}$ we neither accounted for these effects nor the related costs. Since pneumococcal illnesses are more common in children in economically poor sub-Saharan African countries ${ }^{[23-25,28,38]}$ it was assumed that it is a less of a health problem at later ages of life or it can at least be more easily treated at those ages. We are however cognisant of adulthood pneumonia particularly when approaching old age. ${ }^{[39]}$ While some patients will be tested accordingly prior to treatments, a lot more will be given treatments without being given laboratory (X-ray) tests particularly at the health centres or dispensary levels, in which case our treatment cost may be a bit on the high side. Ambulatory related expenses and treatment costs of long-term sequelae have not been taken into account in the model.

Despite the shortcomings listed above the results of this study are very much in line with earlier analyses of pneumococcal vaccination in developing countries. ${ }^{[40]}$ Unfortunately not many cost-effectiveness studies on PCV from low and middle-income countries are available. ${ }^{[4]}$ Chaiyakunapruk et al..${ }^{[42]}$ who have updated the published literature on the cost effectiveness of PCV worldwide, identified that there is still a lack of cost-effectiveness studies from developing countries.

Kim et al. ${ }^{[37]}$ used the PVT epidemiological data to do an economic evaluation of pneumococcal conjugate vaccine using The Gambia setting. Their results showed the vaccine to be cost effective in preventing 630 hospitalisations, 40 deaths and 1000 DALYs over 5 years of life of a birth cohort. There are differences between this paper and that of Kim et al. Firstly, the treatment data of our static model was obtained through the PVT prospective clinical trial in The Gambia setting; secondly, our analysis is from a provider perspective unlike the social perspective-based model of Kim et al.; $;^{[37]}$ thirdly, Kim et al. used an assumed price of US\$3.5 $5^{[38]}$ and their diagnostic and treatment cost estimates were based on cost data from Kenya; $;{ }^{[39]}$ fourthly, our population cohort is 32544 compared to 60000 used by Kim et al.

\section{Conclusions}

This study supports the introduction of nine-valent pneumococcal conjugate vaccine into The Gambia's routine infant vaccination programme. The results demonstrate potential merits in terms of morbidity, mortality, permanent disabilities associated with childhood bacterial pneumonia illnesses, DALYs that could be averted and treatment cost-savings with the use of the vaccine in The Gambia's national infant immunisation programme. The high contribution of pneumococcal infections to paediatric morbidity and mortality in The Gambia, and the effectiveness of the vaccine in reducing all-cause in The Gambian setting, may be factors for making the cost effectiveness of the vaccine particularly compelling.

The study showed that vaccine price is one of the most influential determinants of its cost effectiveness. Whilst there continues to be international donor support (particularly with the establishment of AMC to make expensive new vaccines accessible to low income countries), ${ }^{[30,43]}$ increasing vaccination coverage hence sustenance of necessary EPI infrastructure for those countries should be essential priority areas as well. The average cost of treating pneumonia disease in The Gambia is about US\$191 and the use of the vaccine in 
the national infant immunisation programme will be at an additional cost of US\$274 279 at vaccine price of US\$5.00 per vial in single-dose vials.

Generalisability of this trial based economic evaluation to other countries can be questioned. Not only are there differences in socio-economic characteristics, but there are indeed variations in the distribution of pneumococci serotypes. However, the model used in the analysis makes the results applicable to other developing countries most essentially those in sub-Sahara. Countries must in any case choose those vaccines that include their locally evolving pneumococci serotypes coupled with issues of serotype replacements. With research efforts to cover more and various serotypes in the vaccine by the manufacturer (evidenced in the recent introduction of 13-valent PCV into the market), its benefits to low-income countries like The Gambia would be even more overwhelming. There is however a need for more research to look into issues of serotype replacements and its effect on the impact of the effectiveness of the vaccine.

Though this study and the efficacy trial results ${ }^{[6]}$ indicate highly potential merits from the use of the vaccine, the surveillance programme will certainly provide much further information on the use of the vaccine in real national healthcare situations. We hope that the surveillance programme would also provide information on long-term effects of the vaccine on Gambian children particularly considering the fact that it is a seven-valent vaccine (which is expected to be replaced by a 10 - or 13 -valent vaccine ${ }^{[44]}$ ) that is instead being used. If some sort of cost-effectiveness (economic evaluation) study is incorporated into the surveillance programme, that would provide true pictures and the extent of economic benefits of the use of the vaccine, hence serving as a catalyst for other developing countries to provide the vaccine for their children. It will also help to monitor the incidence of nonvaccine serotypes in the sub-Saharan context.

\section{Declarations}

\section{Conflict of interest}

The Authors declare that they have no conflicts of interest to disclose.

\section{Funding}

This research received no specific grant from any funding agency in the public, commercial or not-for-profit sectors.

\section{Acknowledgements}

The GG/MRC Pneumococcal Vaccine Trial project was supported by a consortium of international donors including the UK Medical Research Council (MRC), World Health Organization (WHO), National Institutes of Health of the United States, Bill and Melinda Gates Foundation, United States Agency for International Development and Wyeth Lerdelle who manufactured and supplied the vaccine.

From The Gambia several people provided relevant data required for the model. These include: Mr Kebba Gibba (National EPI Manager), Mr Baboucarr Boye (EPI Logistics Officer), Mr Sidat Fofana (EPI Communications Officer), Mr John Harris (Purchasing Manager, MRC), Mr Ansumana
Darboe (Financial Accountant, MRC), Dr Claire Oluwalana (Paediatrician, MRC) and Mr Momodou Sanyang (Purchasing Associate, UNICEF-Gambia Office). Mr Alieu Ngum now the Executive Chairperson of the National Planning Commission of The Gambia provided advice from policy makers perspective and in the perspective of national development priorities. Dr Maria Raikou (Research Fellow, London School of Economics and Political Science, UK) provided immense advice regarding the best approach and methodology for the analysis. Through PneumoAdip, Dr Anushua Sinha provided relevant information required for the model as well.

Professor Felicity T Cutts was the Director and Principal Investigator of the trial. She was the architect of the economic evaluation proposal and continued to provide guidance and support (financial and moral) in designing the economic evaluation study and in carrying out the analysis. Drs Godwin Enwere and Akramuz Zaman helped in combining the various data sets for the analysis and clarifying various clinical and epidemiological variables.

The views expressed in this paper are the views of the individual authors and not the institutions they represent. Raymond Hutubessy is a staff member of the WHO. He is responsible for the views expressed in this publication and they do not necessarily represent the decisions, policy or views of the WHO.

\section{References}

1. World Health Organization. Weekly Epidemiological Record. Geneva: World Health Organization, 2007.

2. O’Dempsey TJ. Pneumococcal disease among children in a rural area of West Africa. Pediatr Infect Dis J 1996; 15: 431-437.

3. Wardlaw T et al. Pneumonia the Forgotten Killer of Children. Geneva: UNICEF and World Health Organization, 2006.

4. Greenwood BM et al. Deaths in infancy and early childhood in a well vaccinated rural West African population. Ann Trop Paediatr 1987; 7: 91-99.

5. WHO. Guidelines for Estimating Costs of Introducing New Vaccines into the National Immunisation System. Geneva: World Health Organization, 2002.

6. Cutts F et al. Efficacy of nine-valent pneumococcal conjugate vaccine against pneumonia and invasive pneumococcal disease in The Gambia: randomised, double-blind, placebo-controlled trial. Lancet 2005; 365: 1139-1146.

7. Ministry of Health and Social Welfare. National Health Policy of The Gambia - Health Is Wealth (2007-2020). Banjul: Ministry of Health and Social Welfare, 2007.

8. Gold MR et al. Cost-Effectiveness in Health and Medicine. New York: Oxford University Press, 1996.

9. Tan Torres Edejer T et al. Making Choices in Health: WHO Guide to Cost Effectiveness. Geneva: World Health Organization, 2003: 318.

10. Drummond MF et al. Methods for the Economic Evaluation of Healthcare Programmes, 3rd edn. Oxford: Oxford University Press, 2005: 396.

11. World Health Organization. Guide on Standardisation of Economic Evaluations of Immunisation Programmes. Vol. WHO/ IVB/08.14. Geneva: World Health Organization, 2008.

12. Gold MR et al. Cost-Effectiveness in Health and Medicine. Vol. 315. New York: Oxford University Press, 1996.

13. International Monetary Fund. World Economic Outlook Database. Washington (DC): International Monetary Fund, 2006. 
14. World Health Organization. Macroeconomics and Health: Investing in Health for Economic Development. Report of the Commission on Macroeconomics and Health. Geneva: World Health Organization Commission on Macroeconomics and Health, 2001.

15. Global Alliance for Vaccines and Immunization. Advance Market Commitments (AMCs). http://www.gavialliance.org (accessed 20 February 2007).

16. World Health Organization. Choosing Interventions that are Cost Effective. http://www.who.int/choice/country/gmb/cost/en/ index.html (accessed 26 August 2006). WHO, c2005.

17. World Health Organization. The GAVI financial sustainability planning process. Immunization financing 2010. http:// www.who.int/immunization_financing/analyses/fsp/process/en/ (accessed 20 July 2010).

18. Fryer JE. International Drug Price Indicator Guide. Management Science for Health. 2006. http://www.msh.org (accessed 15 October 2006)

19. World Health Organization. WHO country co-operation strategy. The Republic of The Gambia 2002-2005. 2002.

20. World Health Organization. WHO and UNICEF Review of National Immunisation Coverage. Geneva: World Health Organization, 2005.

21. Dowell F et al. Mortality from pneumonia ib children in the United States, 1939 through 1996. N Engl J Med 2000; 342: 1399-1407.

22. Swartz MN. Bacterial meningitis - a view of the past 90 years. N Engl J Med 2004; 351: 1826-1828.

23. Kellner J, Low D. Autimicrobial Resistance to Streptococcus pneumonia Infections: clinical and epidemiological trends. $B M J$ 1998; 4: 67-77.

24. Campagne A et al. Epidemiology of bacterial meningitis in Niamey, Niger, 1981-96. Bull World Health Organ 1999; 77: 499-508.

25. Otten M et al. Hib-Paediatric Bacterial Meningitis (Hib-PBM) Surveillance Network. Surveillance Manual. Geneva: World Health Organization, 2001.

26. Lieu TA et al. Projected cost-effectiveness of Pneumococcal conjugate vaccination of healthy infants and young children. J Am Med Assoc 2000; 283: 1460-1468.

27. Pandey $\mathrm{M}$ et al. Reduction in total under-five mortality in western Nepal through community-based antimicrobial treatment of pneumonia. Lancet 1991; 1991: 993-997.

28. Tessa $\mathrm{G}$ et al. Outcome of meningitis caused by Streptococcus pneumonia and Haemophilus influenzae type $b$ in children in The Gambia. Trop Med Int Health 2000; 5: 207-213.

29. Murray CJL, Lopez AD, eds. The Global Burden of Disease. A Comprehensive Assessment of Mortality and Disability from Diseases, Injuries and Risk Factors in 1990 and Projected to
2020. Cambridge, MA: Harvard University Press, 1996: 412418.

30. Global Alliance for Vaccines and Immunisation. Advanced Market Commitments for Vaccines: Pneumococcal AMC process sheet. 2009. http://www.vaccineamc.org/ (accessed 10 October 2009).

31. World Health Organization. Macroeconomics and Health: Investing in Health for Economic Development - Report of the Commission on Macroeconomics and Health. Geneva: World Health Organization, 2001.

32. Pelton SI, Klein JO. The future of pneumococcal conjugate vaccines for prevention of pneumococcal diseases in infants and children. Pediatrics 2002; 110: 805-814.

33. Poehling KA et al. Invasive pneumococcal disease among infants before and after introduction of pneumococcal conjugate vaccine. JAMA 2006; 295: 1668-1674.

34. Jackson LA et al. Effectiveness of pneumococcal polysaccharide vaccine in older adults. $N$ Engl J Med 2003; 348: 1747-1755.

35. Cohen-Poradosu R, Kasper DL. Group a streptococcus epidemiology and vaccine implications. Clin Infect Dis 2007; 45: 863865.

36. Munoz-Almagro $\mathrm{C}$ et al. Emergence of invasive pneumococcal disease caused by nonvaccine serotypes in the era of 7-valent conjugate vaccine. Clin Infect Dis 2008; 46: 174-182.

37. Rivera-Olivero IA et al. Pneumococcal carriage among indegenous warao children in venuzuela: serotypes, susceptibility pattersn, and molecular epidemiology. Clin Infect Dis 2007; 45: $1427-1434$

38. World Health Organization. World Health Statistics. Geneva: World Health Organization, 2006.

39. Sisk J et al. Cost-effectiveness of vaccination against pneumococcal disease among people 50 through 64 years of age: role of comorbid conditions and race. Ann Intern Med 2003; 138: 960968.

40. Sinha A et al. Cost-effectiveness of pneumococcal conjugate vaccination in the prevention of child mortality: an international economic analysis. Lancet 2007; 369: 389-396.

41. Beutels $\mathrm{P}$ et al. Convincing or confusing? Economic evaluations of childhood Pneumococcal conjugate vaccination - a review (2002-2006). Vaccine 2007; 25: 1355-1367.

42. Chaiyakunapruk $\mathrm{N}$ et al. Cost effectiveness of pediatric pneumococcal conjugate vaccines: a comparative assessment of decision-making tools. BMC Med [online] 2011; 9: 53.

43. Global Alliance for Vaccines and Immunisation. Advance Market Commitments (AMCs). 2007. http://www.gavialliance. org (accessed 20 February 2007).

44. PneuADIP. Surveillance and Research Report. Baltimore, MD: John Hopkins Bloomberg School of Public Health, John Hopkins University, 2007. 